J. Clin. Chem. Clin. Biochem.

Vol. 23, 1985, pp. 343-347

\title{
Enzymuria of the Rat: Biorhythms and Sex Differences
}

\author{
By H. Grötsch, M. Hropot, E. Klaus, V. Malerczyk
}

Hoechst AG, Frankfurt/M.-80, FRG and

\section{H. Mattenheimer}

Rush-Presbyterian-St. Luke's Medical Center, Rush Medical College, Department of Biochemistry and Clinical Chemistry Laboratory, Chicago, IL, USA

(Received November 29, 1984/March 21, 1985)

Summary: Alanine aminopeptidase, $\gamma$-glutamyltransferase and $\mathrm{N}$-acetyl- $\beta$ - $D$-glucosaminidase were measured daily over 65 days in 24-hour urine of male and female Wistar rats. The mathematical evaluation was based on the Fourier-analysis. The excretion of alanine aminopeptidase and $\gamma$-glutamyltransferase was higher in male than in female rats. This sex-dependent difference was not observed for $\mathrm{N}$-acetyl- $\beta$ - $D$-glucosaminidase. The excretion of the 3 enzymes followed a biorhythm with a dominant period of 7 days for $\gamma$ glutamyltransferase and $\mathrm{N}$-acetyl- $\beta$ - $D$-glucosaminidase and one of 9 days for alanine aminopeptidase. Biorhythms and sex differences of enzymuria should be considered in experimental designs.

\section{Enzymurie der Ratte: Biorhythmen und Geschlechtsdifferenzen}

Zusammenfassung: Alaninaminopeptidase, $\gamma$-Glutamyltransferase und $\mathrm{N}$-Acetyl- $\beta$ - $D$-glucosaminidase wurden täglich über 65 Tage bei männlichen und weiblichen Wistar-Ratten im $24 \mathrm{~h}$-Urin gemessen. Die Ergebnisse wurden vermittels Fourier-Analyse ausgewertet. Die Ausscheidung von Alaninaminopeptidase und $\gamma$-Glutamyltransferase zeigte deutliche geschlechtsabhängige Unterschiede, wobei von männlichen Ratten mehr ausgeschieden wurde als von weiblichen Tieren. $\mathrm{N}$-Acetyl- $\beta$ - $D$-glucosaminidase zeigt diese Unterschiede nicht. Die Ausscheidung der drei Enzyme unterlag Biorhythmen mit Periodenlängen von sieben Tagen für $\gamma$ Glutamyltransferase und $\mathrm{N}$-Acetyl- $\beta$ - $D$-glucosaminidase und neun Tagen für Alaninaminopeptidase. Biorhythmen und Geschlechtșdifferenzen in der Enzymurie sind bei Versuchsplanungen zu berücksichtigen.

\section{Introduction}

Urine enzymes are being used with increasing frequency as sensitive indicators for early detection of renal damage. Many investigations include a discussion of biorhythms in man and animals, respectively $(1-3)$. A few reports on experimental animal models document this coherence of biorhythms and nephrotoxicity with a close correlation between enzyme excretion and renal damage. Of the large number of urine enzymes, it is mainly those from the proximal tubular cells that are of particular interest for toxicological and clinical investigations. In recent

years the rat has been used as the main experimental animal in enzyme excretion studies. This animal shows significant enzymuria when substances are administered which cause increased enzymuria in man. The rat tolerates well single-cage housing in long term experiments. Enzymes with the smallest experimental variation and good reproducibility are alanine aminopeptidase, $\gamma$-glutamyltransferase and $\mathrm{N}$-acetyl- $\beta-D$-glucosaminidase. The aim of this study was to apply modern mathematical methods to the investigation of biorhythms and sex-specific differences in the excretion of these enzymes over a 
sixty-five day period. Rhythmical changes over the entire sampling period, especially in alanine aminopeptidase and $\gamma$-glutamyltransferase excretion, were already evident from the raw data.

\section{Materials and Methods}

Twenty four-hour urine was collected on 65 consecutive days from 5 female and 5 male Wistar rats with initial mean body weights of $110 \pm 6.1 \mathrm{~g}$ and $118 \pm 5.4 \mathrm{~g}$, respectively. The animals were kept in individual cages and had free access to food and water. Sodium-azide was added to the urine collection vessels as a preservative. The further handling of samples, involving centrifugation, gel filtration and enzyme assays, has already been described (4).

The following computations for calculating the biorhythms were done separately for both sexes:

1. The individual values of the daily excreted enzymes, normalized to body weight, were averaged, calculating the arithmetic means. Statistical analysis of the enzyme activities, creatinine, urine volume and body weight was performed by Student's t-test $(2 \mathrm{p}<0.05)$.

2. The mean values were plotted versus time.

3. For each individual data set linear trends were removed by subtraction of a straight line as obtained by linear regression.

4. The resulting residual sets were submitted to a Fast-FourierTransform $(5,6,7)$.

5. The average spectral densities were plotted versus the possible period lengths, the $\mathrm{X}$-axis being logarithmically divided for clearer visual representation.

\section{Fast-Fourier-Transform}

When studying biorhythms, i. e. analysing some input data for repeating patterns in the time domain, it is reasonable to apply the methods of signal analysis to these time series of measured data. One of these algorithms of mathematical signal analysis is the Fourier-Transform realized on digital computers as FastFourier-Transform. The Fast-Fourier-Transform is a mathematical tool to decompose a digitally represented signal into different contributing frequencies.
We are aware of the fact that the methods of signal analysis are currently the province of mathematicians, electronic engineers or some neuro physiologists. Reference (8) is recommended as introductory reading.

A 'peak' in the plot of spectral density versus period shows that relevant parts of the total 'energy' of a time dependent signal can be expressed by the 'energy' of a sinusoidal waveform with that specific period length, which replicates itself several times over the sampling interval. The sharpness of such a peak is reduced by sampling error and the intrinsic biological variability. Longer recording times increase the number of complete basic cycles, thereby reducing variance and shortening the width of the different peaks. Long-periodical contributions to a power spectrum indicate noise or slow trends in the original signal (in our study period lengths above 16 days).

\section{Results}

The results of the study are shown in tables $1-4$ and in figures 1-6. Daily enzyme output is listed as milliunits against time, and normalized to body weight as $\mathrm{mU} / \mathrm{kg}$. The tables display the mean values and standard deviation of all individual values obtained separately for each sex on day 1, 10, 20, 30, 40,50 and 60 of the observation interval. As can be seen from tables 1 and 3, alanine aminopeptidase and $\gamma$-glutamyltransferase show significant sex-dependent differences during the whole study period, with the exception of $\gamma$-glutamyltransferase on day 1 . Towards the end of the observation time, e. g. on days 40 and 50 , when normalized to body weight, $\mathrm{N}$-acetyl- $\beta-D$ glucosaminidase also shows differences between male and female rats. Moreover, a tendency for increased enzymuria, as compared to day one, is discernible throughout the observation period and appears earlier in males than in females (tab. 1). However, when related to body weight, the activity increases are no longer apparent in female animals (tab. 3).

Tab. 1. Excretion of urinary enzymes in Wistar rats (enzymes expressed in mU/24 hours, $n=5 \delta$ and 5 o).

\begin{tabular}{|c|c|c|c|c|c|c|c|c|}
\hline & & Day 1 & Day 10 & Day 20 & Day 30 & Day 40 & Day 50 & Day 60 \\
\hline \multirow[t]{2}{*}{ Alanine aminopeptidase } & 0 & $\begin{array}{r}53 \\
\pm \quad 12\end{array}$ & $\begin{array}{c}\left.142^{2}\right) \\
35\end{array}$ & $\begin{array}{c}\left.190^{2}\right) \\
32\end{array}$ & $\begin{array}{c}\left.254^{2}\right) \\
60\end{array}$ & $\begin{array}{c}\left.302^{2}\right) \\
51\end{array}$ & $\begin{array}{c}\left.165^{2}\right) \\
35\end{array}$ & $\begin{array}{c}\left.310^{2}\right) \\
37\end{array}$ \\
\hline & $q$ & $\begin{array}{r}\left.27^{1}\right) \\
\pm \quad 6\end{array}$ & $\begin{array}{l}\left.48^{1}\right) \\
20\end{array}$ & $\begin{array}{l}\left.\left.46^{1}\right)^{2}\right) \\
12\end{array}$ & $\begin{array}{c}\left.\left.49^{1}\right)^{2}\right) \\
7\end{array}$ & $\begin{array}{l}\left.\left.68^{1}\right)^{2}\right) \\
18\end{array}$ & $\begin{array}{c}\left.\left.38^{1}\right)^{2}\right) \\
8\end{array}$ & $\begin{array}{l}\left.\left.60^{1}\right)^{2}\right) \\
17\end{array}$ \\
\hline \multirow[t]{2}{*}{$\gamma$-Glutamyltransferase } & 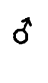 & $\begin{array}{r}905 \\
\pm 246\end{array}$ & $\begin{array}{l}\left.3160^{2}\right) \\
1099\end{array}$ & $\begin{array}{l}\left.4110^{2}\right) \\
1090\end{array}$ & $\begin{array}{l}\left.7164^{2}\right) \\
1528\end{array}$ & $\begin{array}{c}\left.7197^{2}\right) \\
979\end{array}$ & $\begin{array}{c}\left.5125^{2}\right) \\
965\end{array}$ & $\begin{array}{l}8867^{2} \text { ) } \\
1120\end{array}$ \\
\hline & 운 & $\begin{array}{r}784 \\
\pm \quad 294\end{array}$ & $\begin{array}{c}\left.1264^{1}\right) \\
383\end{array}$ & $\begin{array}{c}\left.\left.1387^{1}\right)^{2}\right) \\
318\end{array}$ & $\begin{array}{c}\left.\left.1714^{1}\right)^{2}\right) \\
234\end{array}$ & $\begin{array}{c}\left.\left.2187^{1}\right)^{2}\right) \\
721\end{array}$ & $\begin{array}{c}\left.\left.1656^{1}\right)^{2}\right) \\
365\end{array}$ & $\begin{array}{c}\left.\left.2076^{1}\right)^{2}\right) \\
579\end{array}$ \\
\hline \multirow[t]{2}{*}{$\mathrm{N}$-Acetyl- $\beta$ - $D$-glucosaminidase } & $\delta$ & $\begin{array}{r}77 \\
\pm \quad 39\end{array}$ & $\begin{array}{c}\left.165^{2}\right) \\
29\end{array}$ & $\begin{array}{c}\left.188^{2}\right) \\
42\end{array}$ & $\begin{array}{c}\left.192^{2}\right) \\
64\end{array}$ & $\begin{array}{c}\left.196^{2}\right) \\
62\end{array}$ & $\begin{array}{c}\left.183^{2}\right) \\
.73\end{array}$ & $\begin{array}{c}\left.237^{2}\right) \\
80\end{array}$ \\
\hline & o & $\begin{array}{r}90 \\
\pm \quad 39\end{array}$ & $\begin{array}{c}\left.157^{2}\right) \\
29\end{array}$ & $\begin{array}{c}\left.179^{2}\right) \\
34\end{array}$ & $\begin{array}{c}\left.173^{2}\right) \\
9\end{array}$ & $\begin{array}{c}\left.204^{2}\right) \\
17\end{array}$ & $\begin{array}{c}\left.165^{2}\right) \\
12\end{array}$ & $\begin{array}{c}\left.165^{2}\right) \\
39\end{array}$ \\
\hline
\end{tabular}

1) sex differences tested by Student's t-test (unpaired data), $2 \mathrm{p}<0.05$

$\left.{ }^{2}\right)$ differences versus day 1 tested by Student's t-test (paired data), $2 \mathrm{p}<0.05$ 
Tab. 2. Excretion of urine and creatinine in Wistar rats (urine expressed in ml/24 hours; creatinine expressed in $\mu \mathrm{mol} / 24 \mathrm{hours}$, $n=5 \delta$ and $5 \%$ ).

\begin{tabular}{|c|c|c|c|c|c|c|c|c|}
\hline & & Day 1 & Day 10 & Day 20 & Day 30 & Day 40 & Day 50 & Day 60 \\
\hline \multirow[t]{2}{*}{ Creatinine } & $\sigma$ & $\begin{array}{r}40.4 \\
\pm \quad 4.8\end{array}$ & $\begin{array}{r}47.4 \\
8.1\end{array}$ & $\begin{array}{c}\left.55.6^{2}\right) \\
4.8\end{array}$ & $\begin{array}{l}\left.92.1^{2}\right) \\
15.2^{2}\end{array}$ & $\begin{array}{l}\left.96.6^{2}\right) \\
14.0\end{array}$ & $\begin{array}{l}\left.94.9^{2}\right) \\
18.3\end{array}$ & $\begin{array}{c}\left.133.0^{2}\right) \\
15.4\end{array}$ \\
\hline & q & $\begin{array}{r}37.5 \\
\pm \quad 4.5\end{array}$ & $\begin{array}{c}\left.45.1^{2}\right) \\
5.8\end{array}$ & $\begin{array}{c}\left.51.7^{2}\right) \\
9.5\end{array}$ & $\begin{array}{c}\left.\left.73.3^{1}\right)^{2}\right) \\
3.8\end{array}$ & $\begin{array}{c}\left.\left.74.3^{1}\right)^{2}\right) \\
5.6\end{array}$ & $\begin{array}{c}\left.\left.69.9^{1}\right)^{2}\right) \\
6.2\end{array}$ & $\begin{array}{c}\left.\left.74.6^{1}\right)^{2}\right) \\
8.0\end{array}$ \\
\hline \multirow[t]{2}{*}{ Urine } & $\sigma$ & $\begin{array}{r}7.4 \\
\pm \quad 1.0\end{array}$ & $\begin{array}{l}9.4 \\
2.2\end{array}$ & $\begin{array}{l}9.3 \\
1.4\end{array}$ & $\begin{array}{c}\left.14.5^{2}\right) \\
3.0\end{array}$ & $\begin{array}{c}\left.15.2^{2}\right) \\
3.7\end{array}$ & $\begin{array}{c}\left.12.1^{2}\right) \\
2.3\end{array}$ & $\begin{array}{c}\left.16.6^{2}\right) \\
2.7\end{array}$ \\
\hline & q & $\begin{array}{r}7.2 \\
\pm \quad 1.2\end{array}$ & $\begin{array}{l}7.8 \\
2.7\end{array}$ & $\begin{array}{l}8.7 \\
3.2\end{array}$ & $\begin{array}{l}\left.\left.9.4^{1}\right)^{2}\right) \\
1.8^{2}\end{array}$ & $\begin{array}{r}11.3 \\
2.8\end{array}$ & $\begin{array}{c}\left.11.1^{2}\right) \\
3.1\end{array}$ & $\begin{array}{c}\left.11.3^{1}\right) \\
3.5\end{array}$ \\
\hline
\end{tabular}

1) sex differences tested by Student's t-test (unpaired data), $2 p<0.05$

$\left.{ }^{2}\right)$ differences versus day 1 tested by Student's t-test (paired data), $2 \mathrm{p}<0.05$

Tab. 3. Excretion of urinary enzymes in Wistar rats (enzymes expressed in mU/24 hours $\cdot \mathrm{kg}$ body weight, $\mathrm{n}=5 \delta$ and 5 \%).

\begin{tabular}{|c|c|c|c|c|c|c|c|c|}
\hline & & Day1 & Day 10 & Day 20 & Day 30 & Day 40 & Day 50 & Day 60 \\
\hline \multirow[t]{2}{*}{ Alanine aminopeptidase } & 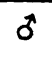 & $\begin{array}{r}448 \\
\pm \quad 85\end{array}$ & $\begin{array}{l}\left.872^{2}\right) \\
252\end{array}$ & $\begin{array}{c}\left.883^{2}\right) \\
93\end{array}$ & $\begin{array}{c}\left.1015^{2}\right) \\
199\end{array}$ & $\begin{array}{l}\left.833^{2}\right) \\
101\end{array}$ & $\begin{array}{l}679 \\
287\end{array}$ & $\begin{array}{l}\left.815^{2}\right) \\
145\end{array}$ \\
\hline & 우 & $\begin{array}{r}\left.245^{1}\right) \\
\pm \quad 69\end{array}$ & $\begin{array}{l}\left.334^{1}\right) \\
130\end{array}$ & $\begin{array}{c}\left.276^{1}\right) \\
74\end{array}$ & $\begin{array}{c}\left.273^{1}\right) \\
41\end{array}$ & $\begin{array}{c}\left.238^{1}\right) \\
41\end{array}$ & $\begin{array}{c}\left.204^{1}\right) \\
70\end{array}$ & $\begin{array}{c}\left.282^{1}\right) \\
61\end{array}$ \\
\hline \multirow[t]{2}{*}{$\gamma$-Glutamyltransferase } & $\delta$ & $\begin{array}{r}7621 \\
\pm 1797\end{array}$ & $\begin{array}{c}\left.19273^{2}\right) \\
7411\end{array}$ & $\begin{array}{c}19186^{2} \\
4470\end{array}$ & $\begin{array}{c}28712^{2} \\
5505\end{array}$ & $\begin{array}{c}\left.24509^{2}\right) \\
3227\end{array}$ & $\begin{array}{c}\left.15331^{2}\right) \\
2249\end{array}$ & $\begin{array}{c}\left.24876^{2}\right) \\
3508\end{array}$ \\
\hline & 우 & $\begin{array}{r}7725 \\
+3078\end{array}$ & $\begin{array}{l}\left.8824^{1}\right) \\
2512\end{array}$ & $\begin{array}{l}\left.8432^{1}\right) \\
2050\end{array}$ & $\begin{array}{l}\left.9628^{1}\right) \\
1439\end{array}$ & $\begin{array}{c}\left.11176^{1}\right) \\
3518\end{array}$ & $\begin{array}{l}\left.8213^{1}\right) \\
1553\end{array}$ & $\begin{array}{l}\left.9935^{1}\right) \\
2286\end{array}$ \\
\hline \multirow[t]{2}{*}{$N$-Acetyl- $\beta$ - $D$-glucosaminidase } & $\delta$ & $\begin{array}{r}647 \\
\pm \quad 333\end{array}$ & $\begin{array}{r}1000 \\
173\end{array}$ & $\begin{array}{l}875 \\
140\end{array}$ & $\begin{array}{l}767 \\
234\end{array}$ & $\begin{array}{l}665 \\
198\end{array}$ & $\begin{array}{l}548 \\
210\end{array}$ & $\begin{array}{l}661 \\
213\end{array}$ \\
\hline & 운 & $\begin{array}{r}816 \\
\pm \quad 350\end{array}$ & $\begin{array}{r}1105 \\
214\end{array}$ & $\begin{array}{r}1089 \\
229\end{array}$ & $\begin{array}{r}972 \\
49\end{array}$ & $\begin{array}{c}\left.1048^{1}\right) \\
99\end{array}$ & $\begin{array}{l}\left.829^{1}\right) \\
102\end{array}$ & $\begin{array}{l}811 \\
228\end{array}$ \\
\hline
\end{tabular}

1) sex differences tested by Student's t-test (unpaired data), $2 \mathrm{p}<0.05$

$\left.{ }^{2}\right)$ differences versus day 1 tested by Student's t-test (paired data), $2 \mathrm{p}<0.05$

Tab.4. Excretion of creatinine and urine and alteration of body weights in Wistar rats (creatinine and urine volumes expressed in $\mu \mathrm{mol}$ resp. $\mathrm{ml} / 24$ hours $\cdot \mathrm{kg}$ body weight, $\mathrm{n}=5 \sigma^{2}$ and 5 \%).

\begin{tabular}{|c|c|c|c|c|c|c|c|c|}
\hline & & Day 1 & Day 10 & Day 20 & Day 30 & Day 40 & Day 50 & Day 60 \\
\hline \multirow[t]{2}{*}{ Creatinine } & $\pi$ & $\begin{array}{r}341 \\
\pm \quad 30\end{array}$ & $\begin{array}{r}287 \\
47\end{array}$ & $\begin{array}{c}\left.261^{2}\right) \\
23\end{array}$ & $\begin{array}{r}369 \\
48\end{array}$ & $\begin{array}{r}328 \\
34\end{array}$ & $\begin{array}{r}284 \\
41\end{array}$ & $\begin{array}{r}371 \\
23\end{array}$ \\
\hline & q & $\begin{array}{r}341 \\
\pm \quad 27\end{array}$ & $\begin{array}{c}\left.316^{2}\right) \\
35\end{array}$ & $\begin{array}{r}314 \\
62\end{array}$ & $\begin{array}{c}\left.412^{2}\right) \\
26\end{array}$ & $\begin{array}{c}\left.\left.382^{1}\right)^{2}\right) \\
37\end{array}$ & $\begin{array}{c}\left.348^{1}\right) \\
22\end{array}$ & $\begin{array}{r}362 \\
22\end{array}$ \\
\hline \multirow[t]{2}{*}{ Urine volumes } & $\delta$ & $\begin{array}{r}50.9 \\
\pm \quad 23.9\end{array}$ & $\begin{array}{l}57.1 \\
13.3\end{array}$ & $\begin{array}{r}43.7 \\
6.3\end{array}$ & $\begin{array}{r}57.8 \\
9.3\end{array}$ & $\begin{array}{l}51.9 \\
14.5\end{array}$ & $\begin{array}{r}36.2 \\
6.4\end{array}$ & $\begin{array}{r}46.3 \\
6.5\end{array}$ \\
\hline & 웅 & $\begin{array}{r}65.9 \\
+\quad 13.4\end{array}$ & $\begin{array}{l}54.8 \\
18.4\end{array}$ & $\begin{array}{l}53.2 \\
21.0\end{array}$ & $\begin{array}{l}52.7 \\
10.5\end{array}$ & $\begin{array}{l}57.7 \\
13.2\end{array}$ & $\begin{array}{l}\left.55.5^{1}\right) \\
15.9\end{array}$ & $\begin{array}{l}54.7 \\
16.9\end{array}$ \\
\hline Body weight (g) & $\sigma^{*}$ & $\begin{array}{r}118.2 \\
\pm \quad 5.4\end{array}$ & $\begin{array}{c}\left.165.2^{2}\right) \\
12.5\end{array}$ & $\begin{array}{c}\left.213.8^{2}\right) \\
14.8\end{array}$ & $\begin{array}{c}\left.249.4^{2}\right) \\
18.5\end{array}$ & $\begin{array}{c}\left.294.4^{2}\right) \\
24.5\end{array}$ & $\begin{array}{c}\left.332.8^{2}\right) \\
27.8\end{array}$ & $\begin{array}{c}\left.357.8^{2}\right) \\
24.3\end{array}$ \\
\hline .. & $q$ & $\begin{array}{r}109.8 \\
\pm \quad 6.1\end{array}$ & $\begin{array}{c}\left.\left.142.6^{1}\right)^{2}\right) \\
4.3\end{array}$ & $\begin{array}{c}\left.\left.165.0^{1}\right)^{2}\right) \\
4.5\end{array}$ & $\begin{array}{c}\left.\left(178.4^{1}\right)^{2}\right) \\
6.4\end{array}$ & $\begin{array}{c}\left.\left.194.8^{1}\right)^{2}\right) \\
12.8\end{array}$ & $\begin{array}{c}\left.\left.200.8^{1}\right)^{2}\right) \\
14.0\end{array}$ & $\begin{array}{l}\left.\left.207.2^{1}\right)^{2}\right) \\
17.6\end{array}$ \\
\hline
\end{tabular}

1) sex differences tested by Student's t-test (unpaired data), $2 \mathrm{p}<0.05$

$\left.{ }^{2}\right)$ differences versus day 1 testẹ by Student's t-test (paired data), $2 \mathrm{p}<0.05$.

Data on urine volume and creatinine excretion related to 24 hours (tab. 2) and to body weight (tab. 4) display an inconclusive pattern of statistical significances.
In figures $1-3$, the daily excretion of the enzymes in $\mathrm{mU} / \mathrm{kg}$ is shown separately for each sex. This graphical representation already suggests repeating patterns that could be interpreted as biological rhythms. 


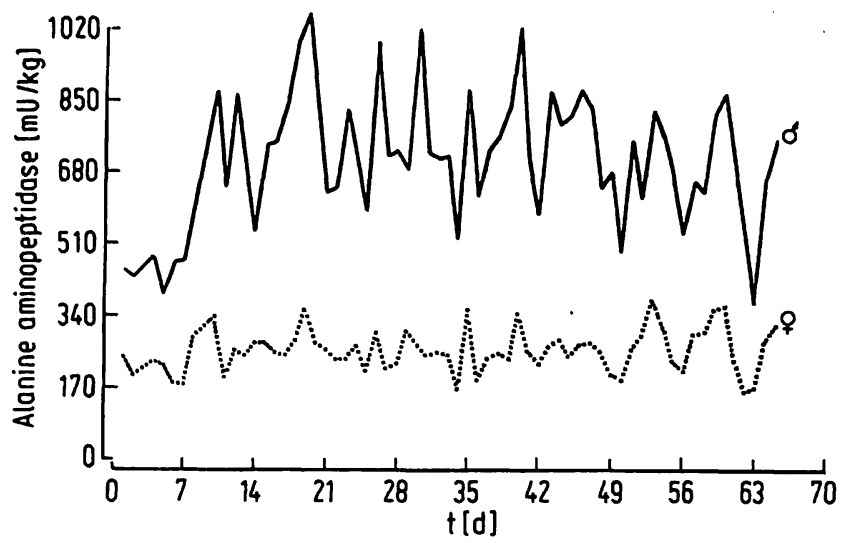

Fig. 1. Urinary excretion of alanine aminopeptidase in 5 male and 5 female rats on 65 consecutive days. Each point represents a mean of 5 values.

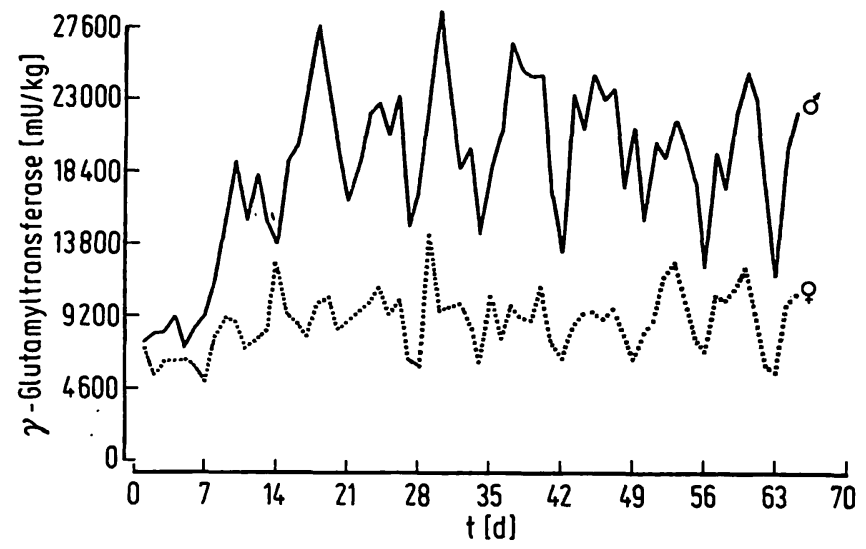

Fig. 2. Urinary excretion of $\gamma$-glutamyltransferase in 5 male and 5 female rats on 65 consecutive days. Each point represents a mean of 5 values.

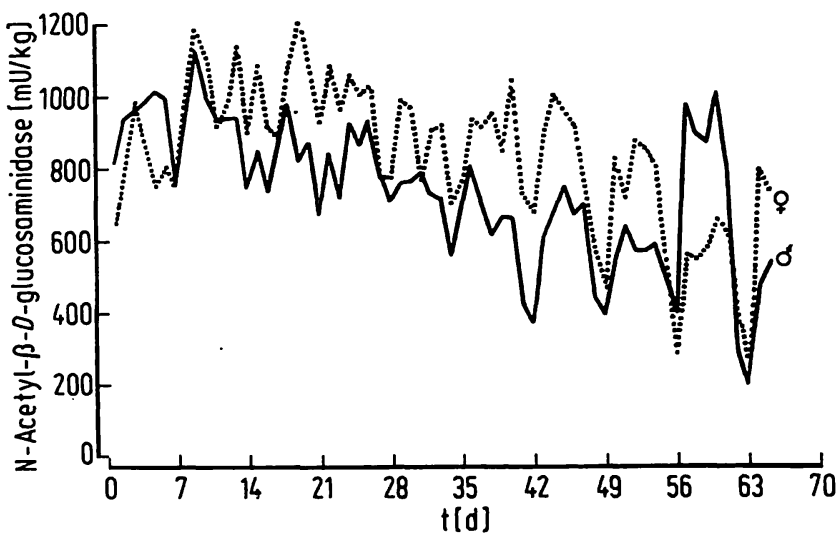

Fig. 3. Urinary excretion of $\mathrm{N}$-acetyl- $\beta$ - $D$-glucosaminidase in 5 male and 5 female rats on 65 consecutive days. Each point represents a mean of 5 values.

The results of the application of the Fast-FourierTransform on the excretion data of each of the recorded enzymes are shown in figures 4-6. At least one maximal peak of spectral density with a frequency of $1 / 7$ cycles/day, i. e. period length equals
7 days, for the enzymes $\gamma$-glutamyltransferase and $\mathrm{N}$ acetyl- $\beta$ - $D$-glucosaminidase and of 9 days for alanine aminopeptidase was observed, thus demonstrating the existence of biorhythms. Additionally all 3 enzymes showed smaller peaks at about 3.5 days being harmonics of the respective basic frequencies. In the $\mathrm{N}$-acetyl- $\beta$ - $D$-glucosaminidase pówer spectrum an additional period was observed at about 5 days, and this was more prominent with female than male rats.

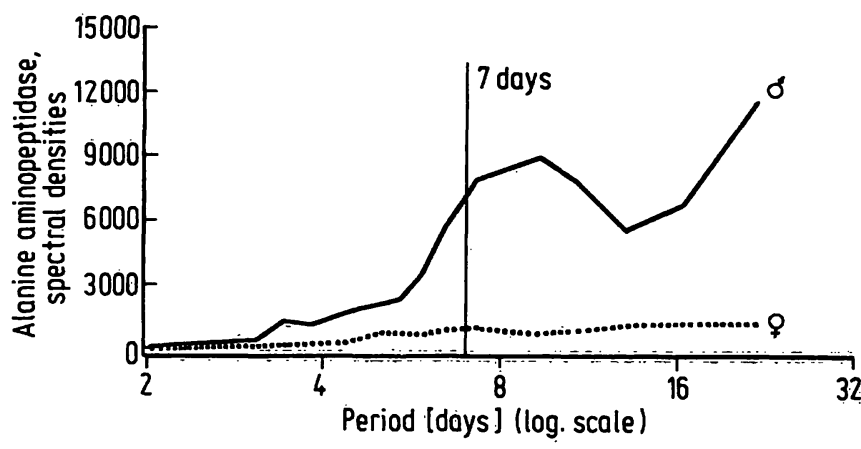

Fig. 4. Spectral densities as obtained by Fast Fourier Transform analysis of long term measurements of alanine aminopeptidase excretion.

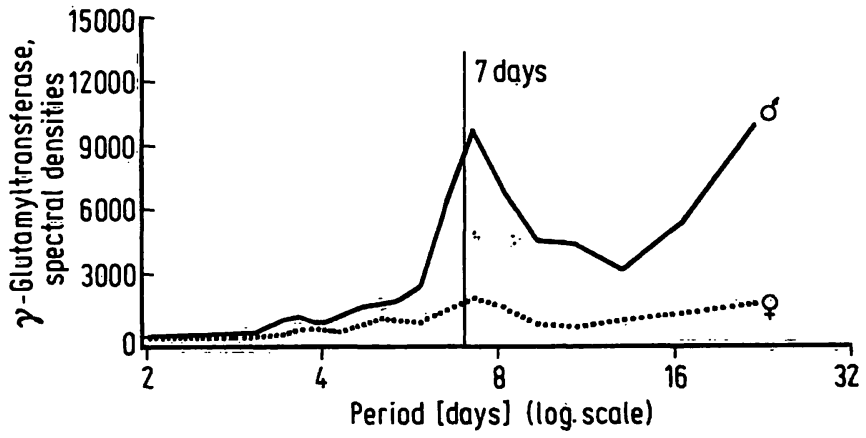

Fig. 5. Spectral densities as obtained by Fast Fourier Transform analysis of long term measurements of $\gamma$ glutamyltransferase excretion.

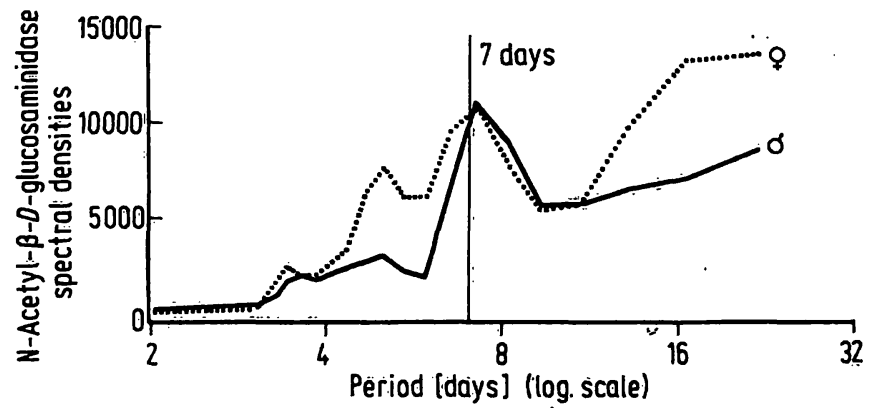

Fig. 6. Spectral densities as obtained by Fast Fourier Transform analysis of long term measurements of $\mathrm{N}$-acetyl- $\beta-D$ glucosaminidase excretion. 


\section{Discussion}

Although excretion of enzymes appears to be a sensitive indicator of renal cell integrity and seems to have diagnostic relevance in the early recognition of kidney diseases, the wide variability of normal values makes it difficult to discriminate between normal and pathological conditions of the kidney (9). In our study the application of the Fast-Fourier-Transform to the recorded enzymes confirms the presence of biorhythms which are already evident in the excretion curves of the enzymes. An explanation for the sexrelated differences could not be found in this study. This varying behaviour of sex-dependent excretion of urinary enzymes as demonstrated in figures $1-3$ was reported for the first time by Grötsch et al. (10) in 1983. These results were confirmed by Simane et al. (11) in 1984. In human volunteers Burchardt \& Miesel (12) also reported sex differences for the excretion of alanine aminopeptidase, males showing higher alanine aminopeptidase-activities than females. On the other hand, Szasz (13) found sex-dependent differences in the activity of $\gamma$-glutamyltransferase, whereas Levy \& Dubach (14) could not reproduce these data. The different ages of volunteers is one possible source of these discrepancies.

An age-related increase of alanine aminopeptidase excretion in man has been reported by Peters et al. (15), whereas Tucker et al. (16) and Wellwood et al. (17) have shown the same pattern for the excretion of $\mathrm{N}$-acetyl- $\beta$ - $D$-glucosaminidase. However, it should be mentioned that these authors calculated the ratio alanine aminopeptidase/creatinine and/or $\mathrm{N}$-acetyl-

\section{References}

1. Burchardt, U., Schinköthe, G., Anton, D., Dreller, E., Neef, L., Ullich, D. \& Patsch, R. (1983) Dt. Gesundh.Wesen 38, 1434-1439.

2. Horpacsy, G. (1979) In: Die Anwendung der Enzymdiagnostik in der Praxis der experimentellen und klinischen Nierentransplantation, F. K. Schattauer Verlag Stuttgart/New York, p p. $16=19$.

3. Lakatua, D. J., Blomquist, C. H., Haus, E., SackettLundeen, L., Berg, H. \& Swoyer, J. (1982) Am. J. Clin. Pathol. 78, 69-77.

4. Berscheid, G., Grötsch, H., Hropot, M., Klaus, E. \& Mattenheimer, H. (1983) J. Clin. Chem. Clin. Biochem. 21, $799-804$.

5. Cooley, J. W. \& Tukey, J. W. (1965) J. Math. Comp., Vol. 19, 297-301.

6. Helwig, J. T. \& Delong, D. M. (1979) SAS User's Guide, pp. $381-388$.

7. Stearns, S. D. (1975) Digital Analysis, Hayden Book Comp., Rochelle Park, N. J. USA.

8. Wiener, N. (1961) In: Cybernetics or control and communication in the animal and the machine, The M.I.T. Press and John Wiley \& Sons, Inc., New York/London, $2^{\text {nd }}$ ed., pp. $181-203$.
$\beta-D$-glucosaminidase/creatinine. Since creatinine excretion in man decreases with age, enzyme/creatinine ratios should be normalized to age if used as reference values. In the present investigation the excretion of enzymes over daily periods was calculated in $\mathrm{mU} /$ $24 \mathrm{~h}$ or related to body weight in $\mathrm{mU} / 24 \mathrm{~h} \cdot \mathrm{kg}$. Although creatinine excretion and body weight correlated equally well in both sexes $(r=0.94)$, we preferred body weight (probably the more notable variable in sex-dependent development) as reference.

In summary we conclude that the rat is an useful animal model for recording urinary enzyme activities over prolonged periods of time. Sex-dependent differences in enzymuria must be taken into account. The urinary excretion of alanine aminopeptidase and $\gamma$-glutamyltransferase increases with age and body weight in male rats, but not in female rats. Baselines of urinary excretion of $\mathrm{N}$-acetyl- $\beta$ - $D$-glucosaminidase and creatinine are comparable during the initial phase of the study. Normalized to body weight, some statistically significant differences between sexes were observed during later stages of the study with respect to $\mathrm{N}$-acetyl- $\beta$ - $D$-glucosaminidase and creatinine. The development of body weight showed sex-specific significant differences. It can also be stated that the excretion of the discussed enzymes shows different forms of chronobiological rhythms, which have been quantified using mathematical methods.

\section{Acknowledgement}

We thank Ms. E. Dichtl and Ms. R. Heun for their skilful assistance.
9. Maruhn, D., Fuchs, I., Mues, G. \& Bock, D. (1976) Clin. Chem. 22, 1567-1574.

10. Grötsch, H., Hropot, M. \& Klaus, E. (1983) Second Joint Meeting of the Belgian, Dutch, German and British Soc. of Clin. Chem., Newcastle upon Tyne, 19. -22. April 1983, poster demonstration and abstracts of papers and posters p. 36.

11. Simane, Z. J. \& Helm, E. (1984) Rev. Brazil. Anal. Clin. 16, 97.

12. Burchardt, U. \& Miesel, B. (1977) Z. Inn. Med. 32, $319-322$.

13. Szasz, G. (1970) Z. Klin. Chem. Klin. Biochem. 8, 1-8.

14. Levy, A. \& Dubach, U. C. (1972) Klin. Wochenschr. 50, $438-441$.

15. Peters, J. E., Schneider, I. \& Haschen, R. J. (1972) Clin. Chim. Acta 36, 289-301.

16. Tucker, S. M., Boyd, P. J. R., Thompson, A. E. \& Price, R. G. (1975) Clin. Chim. Acta 36, 333-339.

17. Wellwood, J. M., Ellis, B. G., Price, R. G., Harmond, K., Thompson, A. E. \& Jones, N. F. (1975) Brit. Med. J. 3, 408-411.

\author{
Dr. Horst Grötsch \\ Hoechst AG \\ D-6230 Frankfurt/M.-80
}


